\title{
Game-theoretical interpretation of abelian logic A
}

\author{
Alexandra Pavlova \\ Higher School of Economics, \\ 20 Myasnitskaya Str., Moscow, 101000, Russian Federation. \\ Saint Petersburg State University, \\ 5 Mendeleevskaya Liniya, St. Petersburg, 199034, Russian Federation. \\ E-mail: pavlova.alex22@gmail.com
}

\begin{abstract}
In the present paper we introduce a variation of Giles's game that captures the semantics of Slaney and Meyer's Abelian logic. This is a variation of the game earlier proposed for the Łukasiewicz infinitely-valued logic. We discuss two possible interpretations of this game. One of the interpretations involves a reference to different types of agents. We also give a brief description of the Abelian logic which as well corresponds to one of the comparative logics proposed by Casari. By different types of agents, we understand agents with diverse cognitive presumptions and capabilities. This reflects the idea that different agents can be encoded by a game (dialogue) semantics and truth (and validity) can be seen as a product of different types of communications between agents, establishing the relation between various types of moves available to the players and the resulting type of rationality. However, the main focus of the paper is concentrated on the technical result concerning the game proposed in the paper. In a separate section, we prove that this game is adequate to the Abelian logic. The game can be extended to the one allowing for the disjunctive strategies. As immediate future research, we suggest proving that Proponent's winning strategies for some formula $F$ in the game for Abelian logic $\mathbf{A}$ with disjunctive strategies correspond to a derivation of the formula $F$ in the hypersequent calculus $\mathbf{G A}$.
\end{abstract}

Keywords: Abelian logic, game semantics, epistemic presumptions, hypersequent calculus, non-classical logic, many-valued logic

For citation: Pavlova A. "Game-theoretical interpretation of abelian logic A", Logicheskie Issledovaniya / Logical Investigations, 2019, Vol. 25, No. 2, pp. 75-93. DOI: 10.21146/20741472-2019-25-2-75-93

\section{Introduction}

In the present paper we introduce a variation of the Giles's game that captures the semantics of the Slaney and Meyer's Abelian logic A Meyer et al., 1989]. The Giles's game is a two-player zero-sum game which can be seen as a valuation game, i.e. semantic game that determines truth in a given model. The model is represented by the risk assignments for each formula. Giles's game is of a particular interest as it combines two types of games: 
- Games determining truth on a model, like Game-Theoretical Semantics of J. Hintikka Hintikka, 1996;

- Lorenzen's dialogue games determining validity [Lorenzen et al., 1978], Krabbe, 2006.

Our article comprises five sections two of them being the introduction and conclusion. In the introduction, we informally show a notion of Giles's game as it was presented for Łukasiewicz infinitely-valued logic. This is done in order to make the initial experiment-based interpretation of the game more evident for a reader. Later we proceed by suggesting our own interpretation of Giles's game for Abelian logic which is much different from the original experiment based interpretation which is due to semantics of Abelian logic. We proceed by presenting the notion of Abelian logic in the section 2. In section 3 the notion of Giles's game for Abelian logic is formally introduced, and finally we prove the adequacy of this game to Abelian logic in section 4 .

To begin with, we present the basic idea of Giles's game for Łukasiewicz infinitely-valued logic $\mathrm{E}$ the way it is presented in Fermüller, 2009 which will later be tailored to capture Abelian logic (see section 3). The game $G([\Gamma \| \Delta], \rho)$ consists of:

- 2 players, i.e. $I$ (me) and $Y$ (you). In our own presentation of the game for Abelian logic we stick to the Lorenzen's names for players, i.e., Proponent for $I$ and Opponent for You. Players bet on formulae by asserting them. As the game proceeds as decompositions of complex formulae, it ends up in an elementary state, i.e., a state where all the formulae asserted by $O$ and $P$ are atomic;

- $\Gamma$ is a multiset that contains all the formulae asserted by $O$ and multiset $\Delta$ contains all the formulae asserted by $P$. Those are multisets because they may contain multiple instances of the same formulae unlike ordinary sets. It is important as each instance of a formula adds up its risk assignment to the sum of the risk undertaken by a player in question;

- the language is used in a restricted version. The language for the logic $\mathrm{E}$ has only one atomic connective $\rightarrow$ and a constant $\perp$ representing falsity. Other connectives can be defined as follows:

$$
\begin{aligned}
& -\neg A=\operatorname{def}_{\text {def }} A \rightarrow \perp \text { (negation), } \\
& -A \& B=\text { def }_{\text {ef }} \neg(A \rightarrow \neg B) \text { (strong conjunction), } \\
& -A \wedge B==_{\text {def }} A \&(A \rightarrow B) \text { (weak conjunction), } \\
& -A \vee B=_{\text {def }}((A \rightarrow B) \rightarrow B) \wedge((B \rightarrow A) \rightarrow A) \text { (disjunction); }
\end{aligned}
$$


- a (repeatable) elementary experiment $E_{q}$ related to some $q$ stating " $E_{q}$ yields a positive result". If $q$ can be either true or false as in classical logic, then the same experiment should lead to the same result over all repetitions. However, if we allow our experiments to exhibit dispersion meaning that the same experiment may yield different results under repetition, then we are not any more in the realm of the classical logic. That means that now the risk associated to asserting each proposition is not a value from the set $\{0,1\}$ but rather a fixed risk value (denoted $\langle q\rangle$ ) in the real unit interval $[0,1]$ is ascribed to any atomic $q$. That yields the game corresponding to the logic $\mathrm{E}$ as proved in [Fermüller, 2009];

- fixed risk value $\langle\cdot\rangle$ in the closed interval $[0,1]$ (for non-classical case) for each propositional variable. $\langle\perp\rangle=1$;

- the risk for a multiset ${ }^{1} p_{1}, p_{2}, \ldots, p_{m}$ is the following: $\left\langle p_{1}, p_{2}, \ldots, p_{m}\right\rangle=$ $\sum_{i=1}^{m}\left\langle p_{i}\right\rangle$;

- $\left(R_{\rightarrow}\right)$ if a user $X$ has asserted $A \rightarrow B$ and another user $Z$ attacks this statement by asserting $A$, the user $X$ has to assert $B$. The user $Z$ can also grant the formula $A \rightarrow B$ asserted by $X$. In the latter case, the granted formula $A \rightarrow B$ is just deleted from the $X$ set of assertions.

\section{Possible Interpretations of the Game}

Giles's game was motivated by the need to account for logical reasoning in physical theories. Atomic statements represent experiments that have fixed probability of a positive outcome. The idea is that players bet on the expectations related to those experiments, i.e. they agree to pay $1 €$ for each incorrect statement. A player asserting the initial formula (whom we call a Proponent) wins a game if they expect no loss of money (i.e., $P$ either pays the same amount to $O$ as $O$ pays to $P$ or $P$ even gets some money from $O$ ).

\section{Resource based interpretation}

Although this interpretation of a game suits well several fuzzy logics, including the Eukasiewicz infinitely-valued logic $\mathrm{E}$, it does not seem to be adequate for the Abelian logic $\mathbf{A}$ since the set of truth values is a proper subset of $\mathbb{R}$ instead of an interval (as for instance in Łukasiewicz infinitely-valued logic $\mathrm{E}$ : $[0,1]$ ). So we suggest another interpretation of the game. We can think of atomic statements (represented by propositional variables) as some sort of special resources that can be both positive and negative, for instance, equity securities (e.g., common stocks) or derivatives. Complex formulae would then represent simple operations on such assets, like addition and subtraction. When a user

\footnotetext{
${ }^{1}$ The notion of a multiset will be formally defined in what follows on page 81
} 
who is trying to show that the formula is true (we will call them the Proponent) in a given model states a complex formula, they state that it's value is nonnegative, i.e. after performing all the operations specified in the formula the Proponent will have a non-negative budget. This interpretation seems to be rather close to one of the possible views on Abelian logic as on the comparative logics proposed by Casari in [Casari, 1989].

\section{Bounded rationality interpretation}

Initially, the game was proposed by Robin Giles in 1970s to model logical reasoning in physics. Thus, Giles's game can be seen as a game related to the knowledge of the expected probability of positive results of experiments. From that follows that the game can be seen as a type of epistemic game, or more precisely a game related to the epistemic states of agents where an epistemic state of an agent is represented truth-functionally. The game characterisation of many-valued logics is also related to our investigations in the area of cognitive presumptions and types of rationality as many-valued logics can be seen as some alternative variants of characterisation of knowledge and degrees of belief [Kubyshkina et al., 2016].

Given the above idea, there can also be an alternative understanding of the Giles's game for Abelian logic, i.e. in terms of bounded agents with respect to their epistemic presumptions. One of the main features of the game for Abelian logic is that there are no versions of the principle of limited liability (sometimes denoted as $L L A$ and $L L D)$ :

Definition 1. Limited liability for attack (LLA): A player $X$ can always decide not to attack an occurrence of a formula that has been asserted by another player $Y$.

Definition 2. Limited liability for defence (LLD): A player $X$ can always assert $\perp$ in reply to an attack by another player $Y$.

As there is no internal weakening in the corresponding hypersequent calculus (cf. [Metcalfe et al., 2005]), thus in the game agents cannot grant formulae (i.e. delete them from the opponents' side), that means that the agents cannot agree on any formula, they need to put all those formulae to the test which represents a very strict understanding of epistemic presuppositions. On the other hand, the absence of internal contraction can be interpreted as agents discussing some factual statements, thus a duplicate of a formula cannot be just added in a run of a game as $q$ being true once, does not grantee it being realised two times. This interpretation is consistent with the first one, it just adds the idea of the rules binding agents' presumptions and capabilities. The detailed study of possible interpretations of Giles's game for $\mathbf{A}$ is subject of a separate research. 


\section{Motivation}

The motivation for the paper is twofold. On the one had, the idea is to suggest a game semantics for Abelian logic to get its dynamic interpretation. And this reveals the connection between proof theory and games with respect to many-valued logics. On the other hand, we are interested in investigating the rational agency and its epistemic presumptions that are implied in the manyvalued logics. We have discussed a related classification of agent types in our previous work [Pavlova, 2017] and this is one of our directions of investigation with respect to that classification. This direction is related to our study of agency and the potential use of games and fuzzy logics to model different types of agents.

\section{Abelian Logic}

In this section we briefly present some of the basic notions with respect to Abelian Logic A. It was introduced by Meyer and Slaney [Meyer et al., 1989] as a logic of relevance. It also coincides with one of the Casari's comparative logics [Casari, 1989] formalising comparisons of majority, minority, and equality in natural languages. There exists a sequent calculus for $\mathbf{A}$ provided by Paoli $[$ Paoli, 2001] as well as a hypersequent calculus.

Definition 3. Let $P$ be a countable set of atomic propositions. The language $\mathcal{L}_{A}$ for Abelian logic is generated by the following BNF:

$$
F::=p|F| F \wedge F|F \vee F| F \rightarrow F|F+F| t,
$$

where $p \in P$.

Negation can be defined as follows: $\neg F=_{\text {def }} F \rightarrow t$. It is also worth mentioning that truth and canonical falsity are identical in this logic: $\neg t=t \rightarrow t$, thus $t=\neg t$.

Definition 4 (Axioms and rules). A is generated by the following schemes of axioms $^{2}$ and rules:

(A1) $A \rightarrow((A \rightarrow B) \rightarrow B)$,

(A2) $(A \rightarrow B) \rightarrow((B \rightarrow C) \rightarrow(A \rightarrow C))$,

(A3a) $A \rightarrow(t \rightarrow A)$,

$(\mathrm{A3b})(t \rightarrow A) \rightarrow A$,

\footnotetext{
${ }^{2}$ Alternatively, we could have used axioms plus substitution rule.
} 


$$
\begin{aligned}
& \text { (A4a) }((A \vee B) \rightarrow C) \rightarrow((A \rightarrow C) \wedge(B \rightarrow C)), \\
& \text { (A4b) }((A \rightarrow C) \wedge(B \rightarrow C)) \rightarrow((A \vee B) \rightarrow C), \\
& \text { (A5a) }((A+B) \rightarrow C) \rightarrow(A \rightarrow(B \rightarrow C)), \\
& \text { (A5b) }(A \rightarrow(B \rightarrow C)) \rightarrow((A+B) \rightarrow C), \\
& \text { (A6) }(A \wedge B) \rightarrow A, \\
& \text { (A7) }(A \wedge B) \rightarrow B, \\
& \text { (A8) }((A \rightarrow B) \wedge(A \rightarrow C)) \rightarrow(A \rightarrow(B \wedge C)), \\
& \text { (A9) }(A \wedge(B \vee C)) \rightarrow((A \wedge B) \vee(A \wedge C)), \\
& \text { (A10) }((A \rightarrow B) \rightarrow B) \rightarrow A,
\end{aligned}
$$

$$
\frac{A \rightarrow B \quad A}{B} M P, \quad \frac{A, B}{A \wedge B} \wedge I .
$$

\section{Some notes on semantics}

As for semantic point of view, the appropriate algebras for $\mathbf{A}$ are latticeordered Abelian groups. There are well-established results with respect to the algebraic semantics for Abelian logic, and we are not going into their details (for the reference, see Metcalfe et al., 2005]). We should only notice once again, that the domain of truth values is the set of real numbers $\mathbb{R}$, whereas the designated value is $\geqslant 0$, i.e., $[0,+\infty)$, and the primitive $t=0$. One should also keep in mind that intensional disjunction and intensional conjunction are identical, which is one of the differences from the $L L$ linear logic.

Definition 5. The assignment function $I$ assigns each propositional variable of the language $\mathcal{L}_{A}$ of Abelian logic a real number: $I:$ Prop $\rightarrow \mathbb{R}$.

Later in section 4, we will need the definition of valuation for our proof of Adequacy of Giles's game for A to Abelian logic. Valuation can be defined as an extension of assignment as follows:

Definition 6. A valuation $v$ for $\mathbf{A}$ is a function from the set of formulae into the set of real numbers $\mathbb{R}\left(v: \mathcal{L}_{A} \rightarrow \mathbb{R}\right)$ that extends an assignment $I$ to propositional variables of values in $\mathbb{R}$ by:

$$
\begin{gathered}
v(t)=0 \\
v(A \rightarrow B)=v(B)-v(A) \\
v(A \vee B)=\min (v(A), v(B)) \\
v(A \wedge B)=\max (v(A), v(B)) \\
v(A+B)=v(A)+v(B)
\end{gathered}
$$


As $\neg A$ is not a primitive as it was defined earlier via $t$ and implication $\left(\neg A=_{\text {def }} A \rightarrow t\right)$, one can deduce the valuation for negation the following way:

$$
v(\neg A)=v(A \rightarrow t)=v(t)-v(A)=0-v(A)=-v(A) .
$$

\section{Giles's Game for Abelian logic A}

In this section we give a formal and precise definition of a Giles's game and highlight some differences between the game for the Eukasiewicz infinitelyvalued logic $\mathrm{E}$ and the one for Abelian logic discussed in the present paper. Those differences can also be interpreted as representing alternative types of agents featuring their cognitive presumptions.

\subsection{The Structure of a Game}

We start by providing the basic definitions with respect to the structure of Giles's Game for logic A. The general idea is that a game start with the initial state which a sequence of formulae asserted by $O$ and $P$ and ends up in the elementary state of the same form containing, however, only atomic propositions. If players start with the initial state and play until the elementary state according to the rules, we would call this a run of a game. Here follows the formal definition.

Definition 7. A run of a game is a sequence of attacks and defences obeying the logical rules and some regulation $\rho$ that begins with the state $[\Pi \| \Sigma]$ a finite (possibly empty) multiset $\Pi$ of formulae that are initially stated by the Opponent (we will use $O$ as a shortening) and a finite (nonempty) multiset $\Sigma$ of formulae that are initially stated by Proponent (here and elsewhere we shall use $P$ ) and ends with an atomic state of the game.

Here we use the notion of a multiset which can be informally described as a modification of the concept of set which allows for multiple instances of the same elements ${ }^{3}$. Ordinary sets are composed of pairwise different elements, i.e., no two elements are the same. As we are making use of multisets here, that means that our $\Pi$ and $\Sigma$ can contain the same formulae multiple times, for instance, we can have the following state: $[p, p, q \| r, q, p, r]$.

\footnotetext{
${ }^{3}$ For the sake of being precise, we put here a formal definition of a multiset (which is not pertinent to the topic of the paper).

Definition 8. Let $S$ be a set. A multiset over $S$ is just a pair $\langle D, f\rangle$, where $D$ is a set and $f: D \rightarrow N$ is a function.
} 


\section{What are the states?}

There are two types of states in the game: $d$-states and $i$-states. We first provide a definition of a d-state (meaning "dialogue state"):

Definition 9. A dialogue state (d-state) of a game is $\left[\begin{array}{lll}\Pi & \| & \Sigma\end{array}\right]$ where $\Pi=\left\{A_{1}, A_{2}, \ldots, A_{m}\right\}$ is a multiset of formulae that are currently asserted by the Opponent and $\Sigma=\left\{B_{1}, B_{2}, \ldots, B_{n}\right\}$ is a multiset of formulae currently asserted by the Proponent.

Each statement can be attacked at most once. We also introduce a notion of $i$-states that that refers to a special state that indicates the active formula of the corresponding $d$-state. Thus, the i-state follows each d-state (except for the atomic one) in a game:

Definition 10. An i-state is an intermediary statement that reflects a player's choice of active formula, i.e. the formula occurrence that gets attacked or defended (like in the rules for disjunction, for instance). These states are the same states as the corresponding d-states $[\Pi \| \Sigma]$ where $\Pi=\left\{A_{1}, A_{2}, \ldots, A_{m}\right\}$ and $\Sigma=\left\{B_{1}, B_{2}, \ldots, B_{n}\right\}$, that also contain a special marking of an active formula denoted by underlining: [ח, $\left.\underline{A_{i}} \| \Sigma, B_{j}\right]$ or $\left[\Pi, A_{i} \| \Sigma, \underline{B_{j}}\right]$.

In our definition of a run of a game (as well as in some other definitions that will follow) we used the notion of an elementary state which contains only atomic propositional variables, but no complex formulae. It is time to give a formal definition to it:

Definition 11. A state $\left[p_{1}, p_{2}, \ldots, p_{m} \| q_{1}, q_{2}, \ldots, q_{n}\right]$ is called elementary (or atomic) if all $p_{i}$ are atomic propositional variables asserted by $O$ and all $q_{j}$ are atomic propositional variables asserted by $P$.

\section{Moving from one state to another}

Now that we know what a state is and what types of states constitute a game (and a run of a game), it is important to specify how players go from one state to another in the most general sense (i.e., regardless of the formulae they asserted). We need to specify the protocol of the game which sometimes referred to as structural rules in the literature (especially on Lorenzen games). And the first question that arises is that of whose move it is at a certain state of a particular game. There is no standard way to determine the sequence of moves, e.g., in Lorenzen's games (or Dialogue Logic) the moves between the Opponent and Proponent alternate whereas in Hintikka game (also known as $G T S$ ) moves are determined by the main connective or the outermost quantifier (or modality). A peculiar feature of Giles's game is that the order of moves does not make any difference, thus there is no unique way to determine the 
sequence of moves. We introduce the notion of regulation $\rho$ which determines whose move it is at a particular state of a game.

Definition 12. A regulation $\rho$ is a function that assigns to each non-elementary d-state either the label $\mathbf{P}$ read as "P initiates the next round and chooses a formula to assert" or the label $\mathbf{O}$, for "O initiates the next round chooses a formula to assert".

The label for an $i$-state should match to the corresponding d-state.

Definition 13. A round consists of the following components:

- Player $\alpha^{4}$ who's turn it is to move according to a particular regulation $\rho$ selects an active formula;

- $\alpha$ either attacks the active formula (by either selecting a subformula that $\beta$ should state or stating a formula itself as in the case of implication) if it is in the $\beta$ 's set of statements or states a formula according to the logical rules if it belongs to $\alpha$ 's own set of statements. $\beta$ should react immediately to each attack, as specified in the following logical rules.

One may notice that the response to an attack (when one is possible according to the logical rules specified below) cannot be postponed. Furthermore, unlike in the Łukasiewicz infinitely-valued logic $\mathrm{E}$, in the game for Abelian logic $\mathbf{A}$, players cannot grant formulae (i.e. delete them from the states without attacking them). This corresponds to the lack of internal weakening in $\mathbf{A}$.

Given the above definitions, we are ready to define the game for $\mathbf{A}$. We distinguish here several rather similar concepts, namely: a game, a game form, and a run of a game. If a run of a game is a precise play that is performed by players, a game form represents all possible strategies (i.e. possible runs of a game) that $O$ and $P$ may have in a game with both fixed initial state $[\Pi \| \Sigma]$ and regulation $\rho$.

Definition 14. A game form $\mathbf{G}([\Pi \| \Sigma], \rho)$ is a tree of starts with the initial d-state $[\Pi \| \Sigma]$ as the root together with all possible successor states $S$ with respect to a particular regulation $\rho$ and the logical rules described below.

A game $\mathcal{G}$ is obtained from the game form by adding a playoff function $\langle\cdot\rangle$ for propositional variables. The playoff function maps all propositional variables to the set of reals $\mathbb{R}$.

\footnotetext{
${ }^{4}$ Variables alpha and $\beta$ refer to arbitrary players s.t. if $\alpha$ is $P$, then $\beta$ is $O$ and visa versa.
} 


\subsection{Logical Rules}

Having defined the general course of the game, we now need to specify the rules for attacks and defences which depend on the logical form of a formula in question. We call them logical rules (though sometimes they are also called particle rules in the literature on logical games).

Definition 15. The Giles's game $\mathfrak{G}_{A}$ for Abelian logic A contains the following logical rules for connectives:

$$
\begin{aligned}
& \begin{array}{cc}
{\left[\Pi \| \Sigma, \underline{A \supset B}^{\mathbf{O}}(R \supset)\right.} & {[\underline{A \supset B}, \Pi \| \Sigma]^{\mathbf{P}}(L \supset)} \\
{[A, \Pi \| \Sigma, B]} & {[B, \Pi \| \Sigma, A]}
\end{array}
\end{aligned}
$$

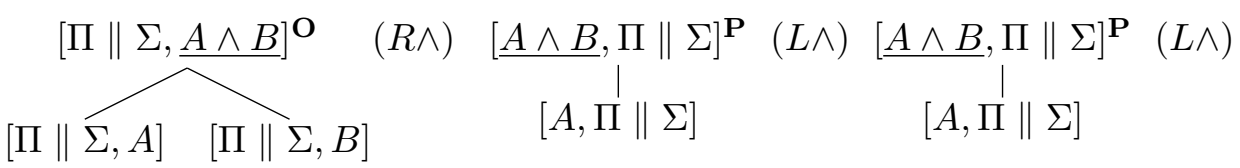

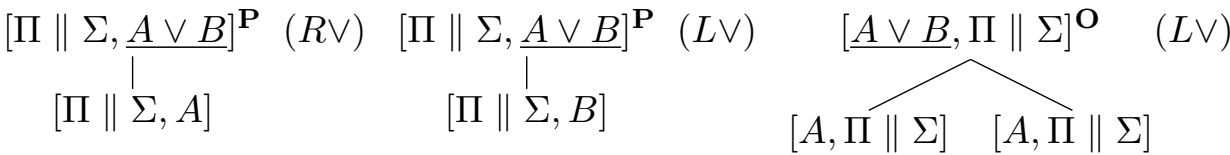

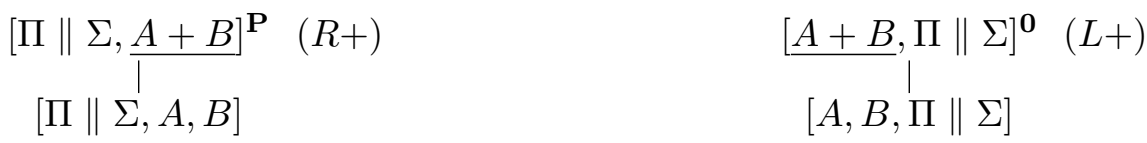

$$
\begin{aligned}
& \begin{array}{cc}
{[\Pi \| \Sigma, t]^{\mathbf{P}}} & (R t) \\
\mid & {[\underline{t}, \Pi \| \Sigma]^{\mathbf{0}}(L t)} \\
{[\Pi \| \Sigma]} & {[\Pi \| \Sigma]}
\end{array}
\end{aligned}
$$

We also introduce a separate rule for truth $t$ which is a special case of the distinguished value for Abelian logic as it coincides with the canonical falsity: $t=\neg t$, i.e., $t=0$. Thus, intensional disjunction and intentional conjunction are the same: $A+B=\neg(\neg A+\neg B)$. Given that negation of a formula is defined the standard way via implication and canonical false, i.e. $\neg A=A \supset t$, one can see that the following rules are admissible (and derivable) given the rules for $t$ and the rules for implication (so for simplicity we add it to our logical rules):

Theorem 1 (The rules for negation).

$$
\begin{gathered}
{[\Pi \| \Sigma, \neg A]^{\boldsymbol{O}}} \\
{[A, \Pi \| \Sigma]}
\end{gathered}
$$

$$
\begin{gathered}
{[\neg A, \Pi \| \Sigma]^{P}} \\
{[\Pi \| \Sigma, A]}
\end{gathered}
$$


Proof. Given the definition of negation as follows $\neg A={ }_{\text {def }} A \supset t$, we derive the rules for $\neg A$ as follows:
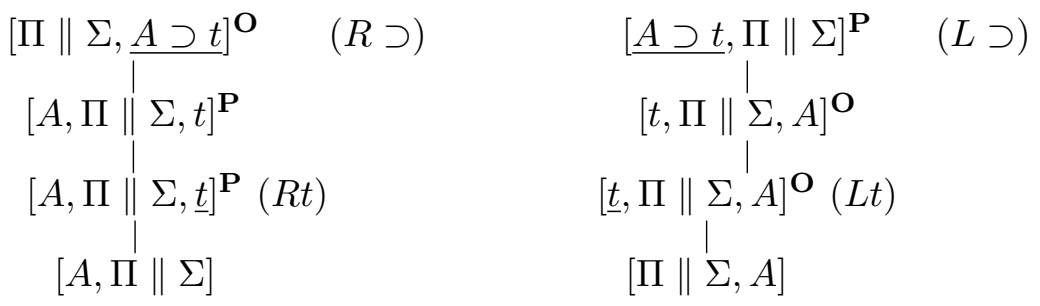

\subsection{Winning Conditions and Strategies}

Before we can define the winning conditions for players, it is necessary to make a few remarks on the playoff of the Giles's game for the Abelian logic. As we have stated before, in the original Giles's game we associate to each atomic variable a risk value $\langle r\rangle$ which can be related to the valuation of the formula in $\mathrm{E}$ as follows: $v(r)=1-\langle r\rangle$. As in the Abelian logic A propositional variables rage over the $\mathbb{R}$, we may directly interpret arbitrary real valued payoffs in a Giles-style game as truth values.

Definition 16. A playoff function $\langle\cdot\rangle$ is a function that assigns each propositional variable a number from the set of real numbers, i.e, $\langle\cdot\rangle=f: \operatorname{Prop} \rightarrow \mathbb{R}$, where Prop is a set of all propositional variables of $\mathcal{L}_{A}$.

Now we are ready to define the winning conditions (abbreviated as w.c.) for $P$ and $O$ in a run of a game with a given regulation $\rho$, and playoff function $\langle\cdot\rangle$.

Definition 17. The Proponent wins in a particular game if the atomic state satisfies the following conditions: $\sum_{i=0}^{m} p_{i} \leqslant \sum_{j=0}^{n} q_{j}{ }^{5}$. Otherwise, the Opponent wins.

However, to define satisfiability and validity via games, we need the concept of a winning strategy for a player in a game. Normally a strategy for a player is defined as a function from states to states that determines a unique choice for that player but includes all the possible actions of the other player (or players if there is more than two). A strategy is subtree of a game $\mathcal{G}([\Pi \| \Sigma], \rho,\langle\cdot\rangle)$ such that:

1. The root node is the $d$-state $[\Pi \| \Sigma]$;

2. All leaf nodes are elementary d-states;

\footnotetext{
${ }^{5}$ Note that the elementary state $[\Pi \| \Sigma]$ can be empty (or have $\Pi=\emptyset$ or $\Sigma=\emptyset$ ) in particular cases of games on the formulae containing $t$, e.g. $\mathbf{G}([t \| t], \rho)$ for some arbitrary $\rho$.
} 
3. If for some node $\left[\Pi_{i} \| \Sigma_{j}\right]$ in the tree $\rho\left(\left[\Pi_{i} \| \Sigma_{j}\right]\right)=P$, then $\left[\Pi_{i} \| \Sigma_{j}\right]$ has exactly one successor $i$-state which has only one successor $d$-state which is the choice of the Proponent with respect to the logical rules;

4. If for some node $\left[\Pi_{i} \| \Sigma_{j}\right]$ in the tree $\rho\left(\left[\Pi_{i} \| \Sigma_{j}\right]\right)=O$, then $\left[\Pi_{i} \| \Sigma_{j}\right]$ has a set of all possible successor $i$-states representing the possible marking. These $i$-states has a set of successor $d$-states which represent all possible choices of the Opponent with respect to the logical rules;

Definition 18 (Winning Strategy). A finite game tree $T$ is a winning strategy $\tau$ for $P$ with respect to a particular playoff function $\langle\cdot\rangle$ iff each leave (of the tree representing the strategy) ends with the elementary state $\left[p_{a}, p_{2}, \ldots, p_{m} \| q_{1}, q_{2}, \ldots, q_{n}\right]$ satisfying the condition $(17): \sum_{i=0}^{m} p_{i} \leqslant \sum_{j=0}^{n} q_{j}$ where $\sum_{j=0}^{n} q_{j}=\left\langle q_{1}, q_{2}, \ldots, q_{n}\right\rangle$ and $\sum_{i=0}^{m} p_{i}=\left\langle p_{a}, p_{2}, \ldots, p_{m}\right\rangle^{6}$.

We shall define the playoff $\langle\cdot \| \cdot\rangle$ of the run of a game $\mathcal{G}([\Pi \| \Gamma\rangle], \rho,\langle\cdot\rangle)$ that ends with the elementary state $\left\langle p_{a}, p_{2}, \ldots, p_{m} \| q_{1}, q_{2}, \ldots, q_{n}\right\rangle$ as follows:

$$
\langle\Pi \| \Gamma\rangle=\sum_{j=0}^{n} q_{j}-\sum_{i=0}^{m} p_{i}
$$

\section{Adequacy of Giles's Games $\mathfrak{G}_{A}$ to the Abelain logic A}

In this subsection we establish the relation between the existence of a winning strategy for the Proponent and validity. However, there are several preliminary steps that should be made before we come to the main theorem of the paper.

\section{Auxiliary remarks}

Fist of all, we need also to extend the semantics of $\mathbf{A}$ from formulae to multisets $\Gamma$ of formulae as follows (henceforward $F \in \mathcal{L}_{A}$, i.e., is an arbitrary formula of the above defined language for $\mathbf{A}$ ):

$$
v(\Gamma)=\operatorname{def} \sum_{F \in \Gamma} v(F)
$$

Secondly, we should establish the correspondence between the playoffs in the game and valuation in Abelian logic. Unlike Łukasiwicz logic, here the correspondence is direct, i.e., we use the following mapping:

$$
\langle p\rangle^{v}=v(p) .
$$

\footnotetext{
${ }^{6}$ That means that each elementary state should be as follows with respect to risk assignment $\left\langle q_{1}, q_{2}, \ldots, q_{n}\right\rangle \geqslant\left\langle p_{a}, p_{2}, \ldots, p_{m}\right\rangle$.
} 
The mapping $\langle p\rangle^{v}=v(p)$ which places playoff value assignments in one-to-one correspondence with truth value assignments can be extended to:

$$
\left\langle p_{a}, p_{2}, \ldots, p_{m} \| q_{1}, q_{2}, \ldots, q_{n}\right\rangle^{v}=v\left(\left[q_{1}, q_{2}, \ldots, q_{n}\right]\right)-v\left(\left[p_{a}, p_{2}, \ldots, p_{m}\right]\right) .
$$

Correspondingly, we define the following function for arbitrary states:

$$
\langle\Pi \| \Sigma\rangle^{v}=v(\Sigma)-v(\Pi) .
$$

Finally, note that

$$
v(F)=v([F]) \geqslant 0 \text { iff }\langle\| F\rangle^{v} \geqslant 0 .
$$

Thus, we prove the following theorem:

Theorem 2. A formula $F$ is valid in A iff there exists a winning strategy for Proponent in the game $\mathcal{G}([\| F], p,\langle\cdot\rangle)$ with any playoff assignment $\langle\cdot\rangle$ and with respect to an arbitrary consistent regulation $\rho$.

Proof. To prove this theorem, we are going to use induction on the complexity of states. First, we observe that each game $\mathcal{G}$ is a finite tree ending up with elementary states. Thus, a winning strategy for $P$ is a finite tree ending with elementary states satisfying the winning conditions for $P$, i.e. $\sum_{j=0}^{m} q_{j} \geqslant \sum_{i=0}^{n}$. Given the playoffs $\langle\cdot\rangle$ for propositional variables, we can construct an optimal strategy for $P$ which would maximize the playoff of $P$ (and the playoff of the game the way we defined it in the section 3) as follows:

1. For any choice of the Opponent at a state $S$, we take the minimal playoff (for $P$ ) of all successor states $S^{\prime}$ that follow from the state $S$;

2. For a state $S$ where it is the Proponent's choice, we take the maximal possible playoff (for $P$ ) of possible successor states $S^{\prime}$ that follow the state $S$

Now we have to show that the notion of the maximal playoff can be extended from the elementary states $\left[p_{a}, p_{2}, \ldots, p_{m} \| q_{1}, q_{2}, \ldots, q_{n}\right]$ to arbitrary states of the form $\left[\Pi_{i} \| \Sigma_{j}\right]$ such that the following conditions are satisfied:

$$
\begin{gathered}
\langle\Pi \| \Sigma, A \supset B\rangle=\langle A, \Pi \| \Sigma, B\rangle \\
\langle A \supset B, \Pi \| \Sigma\rangle=\langle A, \Pi \| \Sigma, B\rangle \\
\langle\Pi \| \Sigma, A \vee B\rangle=\max (\langle\Pi \| \Sigma, A\rangle,\langle\Pi \| \Sigma, B\rangle)
\end{gathered}
$$




$$
\begin{gathered}
\langle A \vee B, \Pi \| \Sigma\rangle=\min (\langle A, \Pi \| \Sigma\rangle,\langle B, \Pi \| \Sigma\rangle) \\
\langle\Pi \| \Sigma, A \wedge B\rangle=\min (\langle\Pi \| \Sigma, A\rangle,\langle\Pi \| \Sigma, B\rangle) \\
\langle A \wedge B, \Pi \| \Sigma\rangle=\max (\langle A, \Pi \| \Sigma\rangle,\langle B, \Pi \| \Sigma\rangle) \\
\langle\Pi \| \Sigma, A+B\rangle=\langle\Pi \| \Sigma, A, B\rangle \\
\langle A+B, \Pi \| \Sigma\rangle=\langle A, B, \Pi \| \Sigma\rangle \\
\langle\Pi \| \Sigma, t\rangle=\langle\Pi \| \Sigma\rangle \\
\langle t, \Pi \| \Sigma\rangle=\langle\Pi \| \Sigma\rangle \\
\langle\Pi \| \Sigma, \neg A\rangle=\langle A, \Pi \| \Sigma\rangle \\
\langle\neg A, \Pi \| \Sigma\rangle=\langle A, \Pi \| \Sigma\rangle
\end{gathered}
$$

Now we check that $\langle\Pi \| \Gamma\rangle$ is well-defined, i.e. the above conditions 112 together with the definition of the Proponent's playoff for elementary states can be simultaneously fulfilled. We have to show that $\langle\cdot \| \cdot\rangle$ indeed specifies $P^{\prime} s$ playoff with respect to his optimal game strategy. We proceed by induction on the complexity of the obtained states. The base cases are obvious. For the equation 1, we consider the following induction step:

(1): $\langle\Pi \| \Sigma, A \supset B\rangle=$ $v(\Sigma)-v(\Pi)+v(A \supset B)=$ $v(\Sigma)-v(\Pi)+v(B)-v(A)=$ $\langle\Pi \| \Sigma\rangle+v(B)-v(A)=$ $\langle A, \Pi \| \Sigma, B\rangle$
(2): $\langle A \supset B, \Pi \| \Sigma\rangle=$ $v(\Sigma)-v(\Pi)-v(A \supset B)=$ $v(\Sigma)-v(\Pi)-v(B)+v(A)=$ $\langle\Pi \| \Sigma\rangle-v(B)+v(A)=$ $\langle B, \Pi \| \Sigma, A\rangle$

It is straightforward to check other connectives, so we obtain the following corresponding induction steps: 
(3): $\langle\Pi \| \Sigma, A \vee B\rangle=$ $v(\Sigma)-v(\Pi)+v(A \vee B)=$ $v(\Sigma)-v(\Pi)+\max (v(A), v(B))=$ $\langle\Pi \| \Sigma\rangle+\max (v(A), v(B))=$ $\max (\langle\Pi \| \Sigma, A\rangle,\langle\Pi \| \Sigma, B\rangle)$
(4): $\langle A \vee B, \Pi \| \Sigma\rangle=$ $v(\Sigma)-v(\Pi)-v(A \vee B)=$ $v(\Sigma)-v(\Pi)-\max (v(A), v(B))=$ $\langle\Pi \| \Sigma\rangle+\min (-v(A),-v(B))=$ $\min (\langle\Pi \| \Sigma\rangle-v(A),\langle\Pi \| \Sigma\rangle-v(B))=$ $\min (\langle A, \Pi \| \Sigma\rangle,\langle B, \Pi \| \Sigma\rangle)$

(5): $\langle\Pi \| \Sigma, A \wedge B\rangle=$ $v(\Sigma)-v(\Pi)+v(A \wedge B)=$ (6): $\langle A \wedge B, \Pi \| \Sigma\rangle=$ $v(\Sigma)-v(\Pi)+\min (v(A), v(B))=$ $\langle\Pi \| \Sigma\rangle+\min (v(A), v(B))=$ $v(\Sigma)-v(\Pi)-v(A \wedge B)=$ $v(\Sigma)-v(\Pi)-\min (v(A), v(B))=$ $\langle\Pi \| \Sigma\rangle+\max (-v(A),-v(B))=$ $\min (\langle\Pi \| \Sigma\rangle+v(A),\langle\Pi \| \Sigma\rangle+v(B))=$ $\min (\langle\Pi \| \Sigma, A\rangle,\langle\Pi \| \Sigma, B\rangle)$

(7): $\langle\Pi \| \Sigma, A+B\rangle=$ $v(\Sigma)-v(\Pi)+v(A+B)=$ $v(\Sigma)-v(\Pi)+v(A)+v(B)=$ $\langle\Pi \| \Sigma\rangle+v(A)+v(B)=$ $\langle\Pi \| \Sigma, A, B\rangle$

(9): $\langle\Pi \| \Sigma, t\rangle=$ $v(\Sigma)-v(\Pi)+v(t)=$ $v(\Sigma)-v(\Pi)+0=$ $\langle\Pi \| \Sigma\rangle+0=\langle\boldsymbol{\Pi} \| \mathbf{\Sigma}\rangle$
(8): $:\langle A+B, \Pi \| \Sigma\rangle=$ $v(\Sigma)-v(\Pi)-v(A+B)=$ $v(\Sigma)-v(\Pi)-v(A)-v(B)=$ $\langle\Pi \| \Sigma\rangle-v(A)-v(B)=$ $\langle A, B, \Pi \| \Sigma\rangle$

$$
\begin{gathered}
\text { 10): }\langle t, \Pi \| \Sigma\rangle= \\
v(\Sigma)-v(\Pi)-v(t)= \\
v(\Sigma)-v(\Pi)-0= \\
\langle\Pi \| \Sigma\rangle-0=\langle\boldsymbol{\Pi} \| \mathbf{\Sigma}\rangle
\end{gathered}
$$

And finally the supplementary case for negation:

(11): $\langle\Pi \| \Sigma, \neg A\rangle=$ $v(\Sigma)-v(\Pi)+v(\neg A)=$ $\langle\Pi \| \Sigma\rangle-v(A)=\langle\boldsymbol{A}, \boldsymbol{\Pi} \| \mathbf{\Sigma}\rangle$
(12): $\langle\neg A, \Pi \| \Sigma\rangle=$ $v(\Sigma)-v(\Pi)-v(\neg A)=$ $\langle\Pi \| \Sigma\rangle+v(A)=\langle\boldsymbol{\Pi} \| \boldsymbol{\Sigma}, \boldsymbol{A}\rangle$

Given the correctness of the statements 112 , we prove that our formula $F \geqslant 0$ given a certain valuation $v$ iff $P$ has a winning strategy in a game over $F$ (formally $\mathcal{G}([\Pi \| \Gamma, F], \rho,\langle\cdot\rangle)$, where $\Pi=\Gamma=\emptyset$, i.t. $\mathcal{G}([\| F], \rho,\langle\cdot\rangle)$ ) with the playoff function $\langle\cdot\rangle=v$ and arbitrary regulation $\rho$.

$\Rightarrow$-direction:Assume that $F \geqslant 0$. We proceed by induction on the length of the winning strategy in a game over $F$. Assume that $F$ is a game of $n$ moves, and $P$ has a winning strategy in a game of $n-1$ moves:

1. $F=p$ (is atomic). Then we automatically have that $p \geqslant 0$, thus $P$ wins in the first move, so she has a winning strategy; 
2. $F=A \supset B$. Then, the next state has the following form: $[A \| B]$. By induction hypothesis $P$ has a winning strategy for the state $[A \| B]$, thus $B \geqslant A$. By according to the statement 1. $\langle\Pi \| \Sigma, A \supset B\rangle=\langle A, \Pi \|$ $\Sigma, B\rangle$, thus $P$ still has the strategy for the state $A \supset B$;

3. $F=A \vee B$. Then, the next state has the following one of the following forms: either $[\| A]$ or $[\| B]$. According to the statement 3 , $P$ has a strategy to keep the maximal of the disjunction as it is her choice whether to select $A$ or $B$. By $I H$ (here a later, $I H$ refers to the Induction Hypothesis), $P$ has a w.s. (= winning strategy) for either $[\| A]$ or $[\| B]$, so she has a w.s. for $F$;

4. $F=A \wedge B$. Then according to the logical rules, the next step will be either $[\| A]$ or $[\| B]$. As it is the choice of $O$ and her aim is to minimise the overall playoff for $P$, we should assume that $O$ choses the minimal conjunct. We have proved that $\langle\Pi \| \Sigma, A \wedge B\rangle=\min (\langle\Pi \| \Sigma, A\rangle,\langle\Pi \|$ $\Sigma, B\rangle)$. By our $I H P$ has a w.s. for the game $n-1$ with the initial state being the minimal (w.r.t. to the $P$ 's playoff) of the 2 possible states, namely $[\| A]$ or $[\| B]$;

5. $F=A+B$. Then, the next state is $[\| A, B]$. As we have proved that $\langle\Pi \| \Sigma, A+B\rangle=\langle\Pi \| \Sigma, A, B\rangle$ (statement 7). By $I H, P$ has a w.s. in the game of $n-1$ with the initial state being [\| $A, B]$, so $P$ has a w.s. for the game of $n$;

6. $F=t$. This case is trivial as $t=0$;

7. $F=\neg A$ is just a case of implication: $F=A \supset t$.

$\Rightarrow$-direction:Assume that $P$ has a w.s. in a game over $F$. We proceed by induction on the structure of $F$ :

1. $F=p$ (i.e. it is atomic). Then $P$ has a w.s. iff $\langle\| p\rangle \geqslant 0$. Thus, $v(p) \geqslant 0$;

2. $F=t$. This case is analogous to $\mathrm{n} 1$.

3. $F=A \supset B$. $P$ has a w.s. in a game over $A \supset B$, i.e. $\langle\| A \supset B\rangle \geqslant 0$. By proposition 1. $\langle\| A \supset B\rangle=\langle A \| B\rangle$. By $I H$, as $P$ has a w.s. for $[A \| B]$, then $v(B)-v(A) \geqslant 0$. By definition $v(B)-v(A)=v(A \supset B)$. Thus, $v(A \supset B) \geqslant 0$;

4. $F=A \vee B$. $P$ has $\mathrm{A}$ w.s. in a game over $A \vee B$, i.e. $\langle\| A \vee B\rangle \geqslant 0$. By proposition 3. $\langle\| A \vee B\rangle=\max (\langle\Pi \| \Sigma, A\rangle,\langle\Pi \| \Sigma, B\rangle)$ as it is $P$ who choses a disjunct (thus it is maximum) and $P$ has a w.s. in one of the 
games: either $[\| A]$ or $[\| B]$. Thus, either $\langle\| A\rangle \geqslant 0$ or $\langle\| B\rangle \geqslant 0$. By $I H$, either $v(A) \geqslant 0$ or $v(B) \geqslant 0$. By definition of the valuation function in our semantics, we have $v(A \vee B)=\max (v(A), v(B))$, hence $v(A \vee B) \geqslant 0$;

5. $F=A \wedge B$. $P$ has $\mathrm{A}$ w.s. in a game over $A \wedge B$, i.e. $\langle\| A \wedge B\rangle \geqslant 0$. By proposition 5. $\langle\| A \wedge B\rangle=\min (\langle\Pi \| \Sigma, A\rangle,\langle\Pi \| \Sigma, B\rangle)$ as it is $O$ who choses a conjunct (thus it is minimum), and $P$ has a w.s. in both of the games: [\| $A]$ and $[\| B]$. Thus, both $\langle\| A\rangle \geqslant 0$ and $\langle\| B\rangle \geqslant 0$. By $I H$, both $v(A) \geqslant 0$ and $v(B) \geqslant 0$. By definition of the valuation function in our semantics, we have $v(A \wedge B)=\min (v(A), v(B))$, hence $v(A \wedge B) \geqslant 0$;

6. $A+B . P$ has A w.s. in a game over $A+B$, i.e. $\langle\| A+B\rangle \geqslant 0$. By proposition 7. $\langle\| A+B\rangle=\langle\Pi \| \Sigma, A, B\rangle$ (there is no choice of conjuncts), and $P$ has a w.s. in in a game over $[\| A, B]$. Thus, $\langle\| A, B\rangle \geqslant 0$. By $I H, v(A, B) \geqslant 0$. By the definition of valuation for multisets ${ }^{7}$, we get $v(A, B)=v(A)+v(B)$. But by def. of valuation function in our semantics, we have $v(A+B)=v(A)+v(B)$, hence $v(A+B) \geqslant 0$;

7. $F=\neg A$ is just a case of implication: $F=A \supset t$.

If $v(F) \geqslant 0$, then there is wining strategy for the Proponent for $\mathbf{G}([\| \mid F], \rho)$ with some playoff assignment $\langle\cdot\rangle$ for any consistent regulation $\rho^{8}$. As a formula $F$ is valid iff for any valuation $v$ holds $v(F) \geqslant 0, F \geqslant 0$ for every playoff function $\langle\cdot\rangle$.

As a future path of research, we would like to extend the game for Abelian logic with disjunctive strategies to get a unified strategy for family of games corresponding to one particular formula in question. By a disjunctive strategy we understand the one that allow players to duplicate states, so that there are 2 kinds of non-leaf nodes:

- playing nodes;

- duplicating nodes.

Let us use $D=S_{1} \vee S_{2} \vee \ldots \vee S_{n}$ to denote a state disjunction which can be viewed as a multiset of states because there might be several instances of the same state but the order of the states $\left(S_{1}, S_{2}, \ldots, S_{n}\right)$ is not relevant. We assume the definition of a disjunctive strategy to be the same as in Fermüller, 2009, namely:

${ }^{7} \mathrm{Cf} .4$.

${ }^{8}$ Since 112 do not depend on the order in which they are decomposed. 
Definition 19. "A disjunctive strategy for $D$ respecting a regulation $\rho$ is a tree of state disjunctions with root $D$ where the successor nodes are in principle determined in the same way as for ordinary strategies".

Definition 20. A disjunctive strategy is wining for a player $X$ iff at every leaf node there is at least one component (an elementary d-state) $S_{i}$ of a state disjunction $S_{1} \vee S_{2} \vee \ldots \vee S_{n}$ that is winning for the player $X$.

It is important that for a collection of games on the same formula there exists a disjunctive winning strategy for a player $X$ iff there is an ordinary strategy for every game in that collection.

\section{Conclusion}

In the present paper we have proposed a new game interpretation of the Abelian logic A. This game is based on the Giles's game explaining how reasoning about experiments in physics proceeds. There is a well studied version of the game for the class of Lukasiewicz many-valued logics, including the infinitelyvalued logic $\mathrm{E}$. We have constructed a game for Abelian logic in section 3 and provided two possible interpretations of the game in 1 . The first interpretation suggests that players reason about the resources/costs and budgeting. As the value might be negative, it is natural to think of these assets as securities (i.e., tradable financial assets). This is indeed related to the one of the standard interpretations of $\mathbf{A}$. The second interpretation suggests that the moves that are allowed to agents, represent their cognitive presumptions and the type of their rationality. Both interpretations are subject to our future study in more detail.

The main technical result of the paper is the proof of adequacy of the game to the Abelian logic that is shortly described in a separate section. We have provided a full proof in the section 4 . This gives rise to the future work related to the game that we have proposed.

\section{Future work}

The future work is related to constructing disjunctive strategies for game for Abelian logic and proving the correspondence between hypersequent calculus GA and the game $\mathcal{G}_{A}$, where a derivation in the calculus decodes a winning strategy for Proponent in the game with disjunctive strategies. Furthermore, in the future research our aim will be to analyse the game interpretation of fuzzy logic from the point of view of epistemic and other agents' presumptions taking into account the truth-functional interpretation of epistemic states. We shall also look into possibility of interpreting modal operators in the Giles's game and more generally dialogue game framework to attempt to modal epistemic 
modalities for non-classical logics and information change in this particular game framework.

Acknowledgements. The reported study was funded by RFBR according to the research project № 18-311-00363. The name of the project: "Truth, cognitive agents and information update in non-classical logics".

\section{References}

Casari, 1989 - Casari, E. "Comparative logics and abelian $\ell$-groups", Logic Colloquium 88. Elsevier, 1989, pp. 161-190.

Fermüller, 2009 - Fermüller, C.G. "Giles's Game and the Proof Theory of Łukasiewicz Logic", Studia Logica, 2009, Vol. 92, pp. 27-61.

Girard, 1987 - Girard, J.Y. "Linear logic", in: Theoretical Computer Science, 50, 1987, pp. 1-102.

Hintikka, 1996 - Hintikka, J. The Principles of Mathematics Revisited, Cambridge: Cambridge University Press, 1996.

Krabbe, 2006 - Krabbe, E.C.W. "Dialogue Logic", in: Handbook of the History of Logic, vol. 7, eds. D.M. Gabbay and J. Woods, New York: Elsevier, 2006, pp. 665704 .

Kubyshkina et al., 2016 - Kubyshkina, E., Zaitsev, D.V. "Rational Agency From a Truth-Functional Perspective", in: Logic and Logical Philosophy, 2016, Vol. 25, No. 4, pp. 499-520.

Lorenzen et al., 1978 - Lorenzen, P., Lorenz, K. Dialogische Logik, Darmstadt: Wissenschaftlische Buchgesellschaft, 1978.

Metcalfe et al., 2005 - Metcalfe, G., Olivetti, N., Gabbay, D. "Sequent and Hypersequent Calculi for Abelian and Lukasiewicz Logics", ACM Transactions on Computational Logic (TOCL), 2005, Vol. 6, pp. 578-613.

Meyer et al., 1989 - Meyer, R.K., Slaney, J.K. "Abelian Logic from A to Z", in: Paraconsistent Logic: Essays on the Inconsistent, Philosophia Verlag, ed. G. Priest et al., 1989, pp. 245-288.

Paoli, 2001 - Paoli, F. "Logic and groups", in: Logic and Logical Philosophy, 2001, No. 9, pp. 109-128.

Pavlova, 2017 - Pavlova, A.M. "What Hamblin's Formal Dialectic Tells About the Medieval Logical Disputation", in: Logical Investigations, 2017, Vol. 23, No. 1, pp. 151-176. 